\title{
Nonlinear optimal control for ship propulsion with the use of an induction motor and a drivetrain
}

\author{
Gerasimos Rigatos ${ }^{1, *}$, Krishna Busawon ${ }^{2,}$, Dimitrios Serpanos ${ }^{3,4}$, Vasilios Siadimas ${ }^{3,4,}$, Pier- \\ luigi Siano ${ }^{5}$, and Masoud Abbaszadeh ${ }^{6}$, \\ ${ }^{1}$ Unit of Industrial Automation, Industrial Systems Institute, 26504, Rion Patras, Greece \\ ${ }^{2}$ Nonlinear Control Group, University of Northumbria, Newcastle NE1 8ST, UK \\ ${ }^{3}$ Industrial Systems Institute - RC Athena, 26504, Rion Patras, Greece \\ ${ }^{4}$ Department of Electrical and Computer Engineering, University of Patras, 26504, Rion Patras, Greece \\ ${ }^{5}$ Department of Industrial Engineering, University of Salerno, Fisciano, 84084, Italy \\ ${ }^{6}$ GE Global Research, General Elecric, Niskayuna, 12309, NY, USA
}

\begin{abstract}
A nonlinear optimal (H-infinity) control method is proposed for an electric ship's propulsion system that consists of an induction motor, a drivetrain and a propeller. The control method relies on approximate linearization of the propulsion system's dynamic model using Taylor-series expansion and on the computation of the state-space description's Jacobian matrices. The linearization takes place around a temporary equilibrium which is recomputed at each time-step of the control method. For the approximately linearized model of the ship's propulsion system, an H-infinity (optimal) feedback controller is developed. For the computation of the controller's gains an algebraic Riccati equation is solved at each iteration of the control algorithm. The stability properties of the control method are proven through Lyapunov analysis,
\end{abstract}

\section{Introduction}

Control of the propulsion of electric ships is a non-trivial problem due to the nonlinearities that characterize the associated state-space model and due to the system's functioning under variable conditions [1]-[2]. Aiming at generating high torque, several types of electric motors have been considered, among which induction motors and multi-phase synchronous motors are frequently met [3]-[4]. By considering the dynamics of the drivetrain that transmits motion from the rotor of the electric motor to the propeller, a state-space model of elevated dimensionality is obtained [5]-[6]. The associated control problem, aiming at making the propeller's rotation reach specific reference setpoints is a multi-variable one and receives as inputs the voltages which are applied to the machine's stator. Besides, by considering variable pitch angle at the propeller's blades an additional control input can be generated, allowing for a more dexterous manipulation of the ship's motion [7]-[8]. The results of this article contribute to developing a more efficient control system for an electric ship's propulsion and to managing optimally the associated power electronics [9]-[11].

Actually, in the present article, a nonlinear optimal control approach is developed for the propulsion system of electric ships, comprising a three-phase induction motor, a drivetrain

\footnotetext{
*e-mail: grigat@ieee.org
} 
and a propeller. Nonlinear optimal (H-infinity) control for electric power systems and actuators has been analyzed in [12]-[13]. First, the dynamic model of the propulsion system undergoes approximate linearization around a temporary operating point (equilibrium) which is recomputed at each iteration of the control algorithm. This operating point is defined by the present value of the propulsion system's state vector and the last value of the control inputs vector that was applied to it. The linearization procedure relies on first-order Taylor series expansion of the propulsion system and on the computation of the associated Jacobian matrices [14]-[16]. The modelling error which is induced by the truncation of the higher-order terms in the Taylor series is considered to be a perturbation that is compensated by the robustness of the control algorithm. For the approximately linearized model of the propulsion system an optimal (H-infinity) feedback controller is designed. For the computation of the H-infinity controller's feedback gain an algebraic Riccati equation is repetitively solved at each timestep of the control method [17]. The stability properties of the propulsion system are proven through Lyapunov analysis. First, it is shown that H-infinity tracking performance criterion is satisfied, which signifies elevated robustness against model uncertainty and external disturbances [18]. Next, conditions for the global asymptotic stability of the control scheme are provided.

\section{Dynamic model of the ship propulsion system}

\subsection{Dynamics of the mechanical part}

The propulsion system of the electric ship, comprises a three-phase induction motor, a drivetrain (gearbox), and the propeller (Fig. 1). The rotational motion of the induction motor is given by

$$
J_{m} \frac{d \omega_{m}}{d t}=T_{e}-T_{h s}-B_{m} \omega_{m}
$$

where $T_{e}$ is the electromagnetic torque that is developed by the motor, $T_{h s}$ is the torque developed by the shaft at the motor's side (high-speed), and $B_{m} \omega_{m}$ is a friction torque that opposes to the rotational motion of the rotor. The rotational motion of the ship's propeller is given by

$$
J_{p} \frac{d \omega_{p}}{d t}=T_{l s}-c_{b a} T_{m}-B_{p} \omega_{p}
$$

where $T_{l s}$ is the torque developed by the shaft at the propeller's side (low-speed), $T_{m}$ is the mechanical torque that is exerted on the propeller due to waves and currents, $c_{b a}$ is the variable pitch angle of the propeller's blade, and $B_{p} \omega_{p}$ is a friction torque that opposes to the rotational motion of the propeller.

Considering transmission of motion from the induction motor to the propeller through a drivetrain which comprises a gear of $n_{m}$ teeth at the side of the motor and a gear of $n_{p}$ teeth at the side of the propeller, the relation between the low torque at the motor's side and the high torque at the propeller's side is given by

$$
\frac{T_{h s}}{T_{l s}}=\frac{\omega_{p}}{\omega_{m}}=\frac{n_{m}}{n_{p}} \Rightarrow T_{h s}=\frac{n_{m}}{n_{p}} T_{l s}
$$

The torque of the shaft is due to torsion and at the propeller's side is given by

$$
T_{l s}=K_{1}\left(\theta_{p}-\theta_{m}\right)+D_{1}\left(\omega_{p}-\omega_{m}\right)
$$

where $K_{1}$ is an elasticity coefficient and $D_{1}$ is a damping coefficient. Using that the value of $D_{1}$ is significantly smaller that the value of $K_{1}$ this result into the following relation about the shaft's torque at the propeller's side 


$$
T_{l s}=K_{1}\left(\theta_{p}-\theta_{m}\right)
$$

Consequently, the shaft's torque at the side of the induction motor is given by

$$
T_{h s}=\frac{n_{m}}{n_{p}} K_{1}\left(\theta_{p}-\theta_{m}\right)
$$

Next, about the mechanical part of the transmission system one can define the state variables $x_{1}=\theta_{p}, x_{1}=\omega_{p}, x_{3}=\theta_{m}, x_{4}=\omega_{m}$, and the control input $u_{1}=c_{b a}$. This results into the following state-space description:

$$
\begin{gathered}
\dot{x}_{1}=x_{2} \\
\dot{x}_{2}=\frac{K_{1}}{J_{p}}\left(x_{1}-x_{3}\right)-\frac{B_{p}}{J_{p}} x_{2}-\frac{T_{m}}{J_{p}} u_{1} \\
\dot{x}_{3}=x_{4} \\
\dot{x}_{4}=\frac{n_{m}}{n_{p}} \frac{K_{1}}{J_{m}}\left(x_{1}-x_{3}\right)-\frac{B_{m}}{J_{n}} x_{4}
\end{gathered}
$$

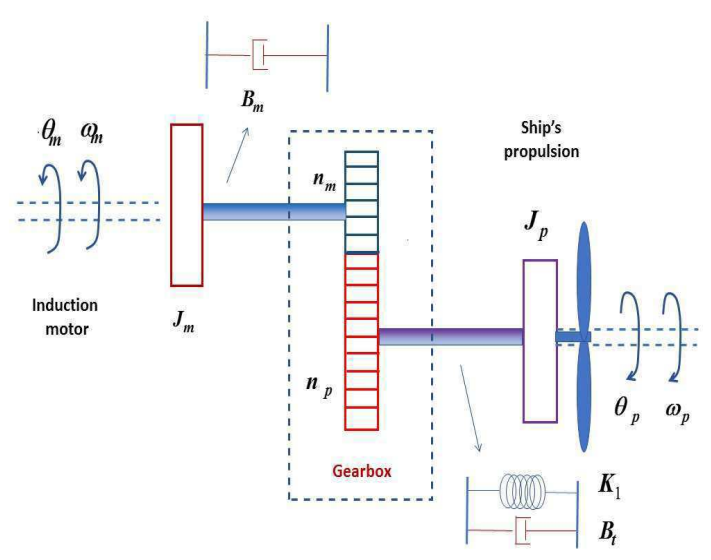

Figure 1. Ship propulsion system comprising an induction motor and a drivetrain

\subsection{Dynamics of the electrical part}

The dynamics of the electrical part of the propulsion system is dependent on the components of the currents of the machine's stator $\left[i_{s d}, i_{s q}\right]$ and on the components of the magnetic flux $\left[\psi_{r d}, \psi_{r q}\right]$, which are expressed in the asynchronously rotating $d q$ reference frame. By applying the field orientation concept, that is by selecting the turn speed of the asynchronously rotating reference frame, defined by the derivative of the angle of the rotor's magnetic field $\rho=\tan ^{-1}\left(\frac{\psi_{r b}}{\psi_{r a}}\right)$ one has that (i) the q-axis component of the magnetic flux vanishes that is $\psi_{r q}=0$, while the d-axis component of the magnetic flux becomes equal to the magnitude of the flux vector, that is $\psi_{r d}=\|\psi\|=\sqrt{\psi_{r a}^{2}+\psi_{r b}^{2}}$, with $\left[\psi_{r a}, \psi_{r b}\right]$ to denote the magnetic flux coefficients in the non-rotating $a b$ reference frame [12]. Under these conditions the dynamics of the electric part of the propulsion system is given by [12] 


$$
\begin{gathered}
\frac{d \psi_{r d}}{d t}=-a \psi_{r d}+a M i_{s d} \\
\frac{d i_{s d}}{d t}=-\gamma i_{s d}+a \beta \psi_{r d}+n_{p} \omega_{m} i_{s q}+\frac{a M i_{s q}^{2}}{\psi_{r d}}+\frac{1}{\sigma L_{s}} v_{s d} \\
\frac{d i_{s q}}{d t}=-\gamma i_{s q}-\beta n_{p} \omega_{m} \psi_{r d}-n_{p} \omega_{m} i_{s d}-\frac{a M i_{s d} i_{s q}}{\psi_{r d}}+\frac{1}{\sigma L_{s}} v_{s q} \\
\frac{d \rho}{d t}=n_{p} \omega_{m}+\frac{a M i_{s q}}{\psi_{r d}}
\end{gathered}
$$

where the model's coefficients are defined as follows: $M$ is the mutual inductance between the stator and the rotor, $L_{s}$ is the stator's inductance, $L_{r}$ is the rotor's inductance, $\sigma=\frac{1-M^{2}}{L_{s} L_{r}}$, $a=\frac{R_{r}}{L_{r}}$ and $\beta=\frac{M}{\sigma L_{s} L_{r}}$. Taking into account the field-orientation condition, the electromagnetic torque that is developed by the induction motor is given by

$$
T_{e}=\mu\left[i_{s q} \psi_{r d}-i_{s d} \psi_{r q}\right] \Rightarrow T_{e}=\mu\left(i_{s q} \psi_{r d}\right)
$$

where coefficient $\mu$ depends on the number of poles of the machine and is defined as $\mu=\frac{n_{p} M}{J_{m} L_{r}}$. Next, by defining the state variables $x_{5}=\psi_{r d}, x_{6}=i_{s d}, x_{7}=i_{s q}$ and $x_{8}=\rho$, where $\rho$ is the orientation angle of the magnetic field (angle between magnetic flux $\psi_{r_{a}}$ and $\psi_{r_{b}}$ ), and the control inputs $u_{2}-v_{s d}$ and $v_{3}=v_{s q}$, one obtains the following state-space description for the electrical part of the propulsion system:

$$
\begin{gathered}
\dot{x}_{5}=-a x_{5}+a M x_{6} \\
\dot{x}_{6}=-\gamma x_{6}+a \beta x_{5}+n_{p} x_{4} x_{7}+\frac{a M x_{7}^{2}}{x_{5}}+\frac{1}{\sigma L_{s}} u_{2} \\
\dot{x}_{7}=-\gamma x_{7}-\beta n_{p} x_{4} x_{5}-n_{p} x_{4} x_{6}-\frac{a M x_{6} x_{7}}{x_{5}}+\frac{1}{\sigma L_{s}} u_{3} \\
\dot{x}_{8}=n_{p} x_{4}+\frac{a M x_{7}}{x_{5}}
\end{gathered}
$$

Moreover, using the previous notation of the state variables the electromagnetic torque which is provided by the motor is given by

$$
T_{e}=\mu x_{5} x_{7}
$$

\subsection{Aggregate dynamics}

By defining the entire state vector of the propulsion system as $x=$ $\left[x_{1}, x_{2}, x_{3}, x_{4}, x_{5}, x_{6}, x_{7}, x_{8}\right]^{T}$ that is $x=\left[\theta_{p}, \omega_{p}, \theta_{m}, \omega_{m}, \psi_{r d}, i_{r d}, i_{s q}, \rho\right]^{T}$, and the entire control inputs vector as $u=\left[u_{1}, u_{2}, u_{3}\right]^{T}$, that is $u=\left[c_{b a}, v_{s d}, v_{s q}\right]^{T}$ one has the complete state-space model

$$
\dot{x}=f(x)+g(x) u
$$

where $x \in R^{8 \times 1}, f(x) \in R^{8 \times 1}, g(x) \in R^{8 \times 3}, u \in R^{3 \times 1}$. In particular $f(x)$ and $g(x)$ are given by:

$$
f(x)=\left(\begin{array}{c}
x_{2} \\
\frac{K_{1}}{J_{p}}\left(x_{1}-x_{3}\right)-\frac{B_{p}}{J_{p}} x_{2} \\
x_{4} \\
\mu x_{5} x_{7}-\frac{1}{J_{m}}\left[\frac{n_{m}}{n_{p}} K_{1}\left(x_{1}-x_{3}\right)-B_{m} x_{4}\right] \\
-a x_{5}+a M x_{6} \\
-\gamma x_{6}+a \beta x_{5}+n_{p} x_{4} x_{7}+\frac{a M x_{7}^{2}}{x_{5}} \\
-\gamma x_{7}-\beta n_{p} x_{4} x_{5}-n_{p} x_{4} x_{6}-\frac{a M x_{6} x_{7}}{x_{5}} \\
n_{p} x_{4}+\frac{a M x_{7}}{x_{5}}
\end{array}\right) \quad g(x)=\left(\begin{array}{ccc}
0 & 0 & 0 \\
-\frac{T_{m}}{J_{p}} & 0 & 0 \\
0 & 0 & 0 \\
0 & 0 & 0 \\
0 & 0 & 0 \\
0 & \frac{1}{\sigma L_{s}} & 0 \\
0 & 0 & \frac{1}{\sigma L_{s}} \\
0 & 0 & 0
\end{array}\right)
$$




\section{Approximate linearization of the ship's propulsion model}

The dynamic model of the propulsion system undergoes approximate linearization at each iteration of the control algorithm, around the temporary operating point $\left(x^{*}, u^{*}\right)$, where $x^{*}$ is the present value of the system's state vector and $u^{*}$ is the last value of the control inputs vector that was applied to it. The linearization relies on first-order Taylor series expansion and on the computation of the associated Jacobian matrices. The linearization procedure results into the state-space description:

$$
\dot{x}=A x+B u+d_{1}
$$

where $d_{1}$ is the modelling error and

$$
\begin{gathered}
A=\left.\nabla_{x}[f(x)+g(x) u]\right|_{\left(x^{*}, u^{*}\right)} \Rightarrow A=\left.\nabla_{x}[f(x)]\right|_{\left(x^{*}, u^{*}\right)} \\
B=\left.\nabla_{u}[f(x)+g(x) u]\right|_{\left(x^{*}, u^{*}\right)} \Rightarrow B=\left.g(x)\right|_{\left(x^{*}, u^{*}\right)}
\end{gathered}
$$

\section{The nonlinear H-infinity control}

\subsection{Tracking error dynamics}

The initial nonlinear model of the electric ship's propulsion system is in the form

$$
\dot{x}=f(x, u) \quad x \in R^{n}, u \in R^{m}
$$

Linearization is performed at each iteration of the control algorithm round its present operating point $\left(x^{*}, u^{*}\right)=\left(x(t), u\left(t-T_{s}\right)\right)$. The linearized equivalent of the induction motor, drivetrain and propeller system is described by

$$
\dot{x}=A x+B u+L \tilde{d} \quad x \in R^{n}, u \in R^{m}, \tilde{d} \in R^{q}
$$

Thus, after linearization round its current operating point, the model of the induction motor, drivetrain and propeller system is written as

$$
\dot{x}=A x+B u+d_{1}
$$

Parameter $d_{1}$ stands for the linearization error in the model of the propulsion system appearing in Eq. (18). The reference setpoints for the propulsion system are denoted by $\mathbf{x}_{\mathbf{d}}=\left[x_{1}^{d}, \cdots, x_{8}^{d}\right]$. Tracking of this trajectory is succeeded after applying the control input $u^{*}$. At every time instant the control input $u^{*}$ is assumed to differ from the control input $u$ appearing in Eq. (18) by an amount equal to $\Delta u$, that is $u^{*}=u+\Delta u$. One can write

$$
\dot{x}_{d}=A x_{d}+B u^{*}+d_{2}
$$

The dynamics of the controlled system described in Eq. (18) can be also written as

$$
\dot{x}=A x+B u+B u^{*}-B u^{*}+d_{1}
$$

and by denoting $d_{3}=-B u^{*}+d_{1}$ as an aggregate disturbance term one obtains

$$
\dot{x}=A x+B u+B u^{*}+d_{3}
$$

By subtracting Eq. (19) from Eq. (21) one has

$$
\dot{x}-\dot{x}_{d}=A\left(x-x_{d}\right)+B u+d_{3}-d_{2}
$$


By denoting the tracking error as $e=x-x_{d}$ and the aggregate disturbance term as $\tilde{d}=d_{3}-d_{2}$, the tracking error dynamics becomes

$$
\dot{e}=A e+B u+\tilde{d}
$$

As noted above, the linearized equivalent of the induction motor, drivetrain and propeller system is described by Eq. (17), where matrices $A$ and $B$ are obtained from the computation of the Jacobians given in Eq. (15). The problem of disturbance rejection for the linearized model that is described by Eq. (17), where $x \in R^{n}, u \in R^{m}, \tilde{d} \in R^{q}$ and $y \in R^{p}$, cannot be handled efficiently if the classical LQR control scheme is applied. The disturbances' effects are incorporated in the following quadratic cost function:

$$
J(t)=\frac{1}{2} \int_{0}^{T}\left[y^{T}(t) y(t)+r u^{T}(t) u(t)-\rho^{2} \tilde{d}^{T}(t) \tilde{d}(t)\right] d t, \quad r, \rho>0
$$

\subsection{H-infinity feedback control}

For the linearized system given by Eq. (17) the cost function of Eq. (24) is defined, where the coefficient $r$ determines the penalization of the control input and the weight coefficient $\rho$ determines the reward of the disturbances' effects. Then, the optimal feedback control law is given by

$$
u(t)=-K x(t)
$$

with $K=\frac{1}{r} B^{T} P$ where $P$ is a positive semi-definite symmetric matrix which is obtained from the solution of the Riccati equation

$$
A^{T} P+P A+Q-P\left(\frac{1}{r} B B^{T}-\frac{1}{2 \rho^{2}} L L^{T}\right) P=0
$$

where $Q$ is also a positive definite symmetric matrix. The worst case disturbance is given by $\tilde{d}(t)=\frac{1}{\rho^{2}} L^{T} P x(t)$. The diagram of the considered control loop is depicted in Fig. 2.

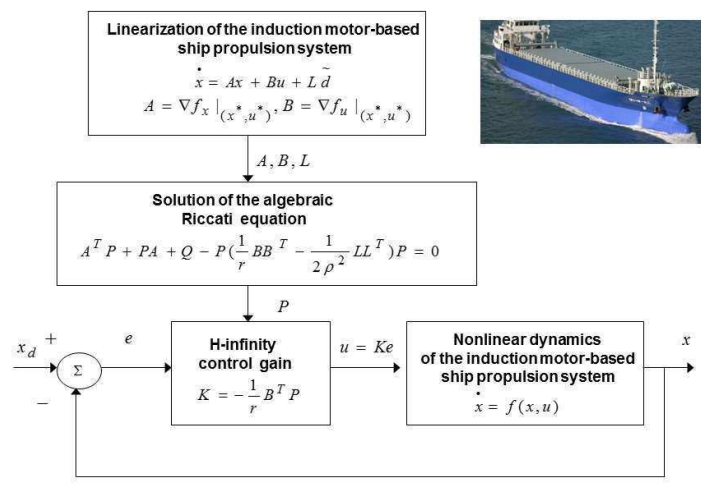

Figure 2. Diagram of the control scheme for the electric ship propulsion system which comprises an induction motor and a drivetrain transferring motion to the propeller 


\section{Lyapunov stability analysis}

The tracking error dynamics for the induction motor, drivetrain and propeller system is written in the form $\dot{e}=A e+B u+L \tilde{d}$, where in the considered power system's case $L=I \in R^{8}$ with $I$ being the identity matrix. Variable $\tilde{d}$ denotes model uncertainties and external disturbances of the model of the ship's propulsion system. The following Lyapunov equation is considered

$$
V=\frac{1}{2} e^{T} P e
$$

where $e=x-x_{d}$ is the tracking error. By differentiating with respect to time one obtains

$$
\begin{gathered}
\dot{V}=\frac{1}{2} e^{T} P e+\frac{1}{2} e^{T} P \dot{e} \Rightarrow \dot{V}=\frac{1}{2}[A e+B u+L \tilde{d}]^{T} P+\frac{1}{2} e^{T} P[A e+B u+L \tilde{d}] \Rightarrow \\
\dot{V}=\frac{1}{2}\left[e^{T} A^{T}+u^{T} B^{T}+\tilde{d}^{T} L^{T}\right] P e+\frac{1}{2} e^{T} P[A e+B u+L \tilde{d}] \Rightarrow \\
\dot{V}=\frac{1}{2} e^{T} A^{T} P e+\frac{1}{2} u^{T} B^{T} P e+\frac{1}{2} \tilde{d}^{T} L^{T} P e+\frac{1}{2} e^{T} P A e+\frac{1}{2} e^{T} P B u+\frac{1}{2} e^{T} P L \tilde{d}
\end{gathered}
$$

The previous equation is rewritten as

$$
\dot{V}=\frac{1}{2} e^{T}\left(A^{T} P+P A\right) e+\left(\frac{1}{2} u^{T} B^{T} P e+\frac{1}{2} e^{T} P B u\right)+\left(\frac{1}{2} \tilde{d}^{T} L^{T} P e+\frac{1}{2} e^{T} P L \tilde{d}\right)
$$

Assumption: For given positive definite matrix $Q$ and coefficients $r$ and $\rho$ there exists a positive definite matrix $P$, which is the solution of the following matrix equation

$$
A^{T} P+P A=-Q+P\left(\frac{2}{r} B B^{T}-\frac{1}{\rho^{2}} L L^{T}\right) P
$$

Moreover, the following feedback control law is applied to the system

$$
u=-\frac{1}{r} B^{T} P e
$$

By substituting Eq. (30) and Eq. (31) one obtains

$$
\begin{gathered}
\dot{V}=\frac{1}{2} e^{T}\left[-Q+P\left(\frac{2}{r} B B^{T}-\frac{1}{\rho^{2}} L L^{T} P\right] e+e^{T} P B\left(-\frac{1}{r} B^{T} P e\right)+e^{T} P L \tilde{d} \Rightarrow\right. \\
\dot{V}=-\frac{1}{2} e^{T} Q e+\left(\frac{1}{r} e^{T} P B B^{T} P e-\frac{1}{2 \rho^{2}} e^{T} P L L^{T} P e-\frac{1}{r} e^{T} P B B^{T} P e\right)+e^{T} P L \tilde{d}
\end{gathered}
$$

which after intermediate operations gives

$$
\begin{gathered}
\dot{V}=-\frac{1}{2} e^{T} Q e-\frac{1}{2 \rho^{2}} e^{T} P L L^{T} P e+e^{T} P L \tilde{d} \Rightarrow \\
\dot{V}=-\frac{1}{2} e^{T} Q e-\frac{1}{2 \rho^{2}} e^{T} P L L^{T} P e+\frac{1}{2} e^{T} P L \tilde{d}+\frac{1}{2} \tilde{d}^{T} L^{T} P e
\end{gathered}
$$

Lemma: The following inequality holds

$$
\frac{1}{2} e^{T} L \tilde{d}+\frac{1}{2} \tilde{d} L^{T} P e-\frac{1}{2 \rho^{2}} e^{T} P L L^{T} P e \leq \frac{1}{2} \rho^{2} \tilde{d}^{T} \tilde{d}
$$

Proof: The binomial $\left(\rho \alpha-\frac{1}{\rho} b\right)^{2}$ is considered. Expanding the left part of the above inequality one gets

$$
\begin{gathered}
\rho^{2} a^{2}+\frac{1}{\rho^{2}} b^{2}-2 a b \geq 0 \Rightarrow \frac{1}{2} \rho^{2} a^{2}+\frac{1}{2 \rho^{2}} b^{2}-a b \geq 0 \Rightarrow \\
a b-\frac{1}{2 \rho^{2}} b^{2} \leq \frac{1}{2} \rho^{2} a^{2} \Rightarrow \frac{1}{2} a b+\frac{1}{2} a b-\frac{1}{2 \rho^{2}} b^{2} \leq \frac{1}{2} \rho^{2} a^{2}
\end{gathered}
$$

The following substitutions are carried out: $a=\tilde{d}$ and $b=e^{T} P L$ and the previous relation becomes

$$
\frac{1}{2} \tilde{d}^{T} L^{T} P e+\frac{1}{2} e^{T} P L \tilde{d}-\frac{1}{2 \rho^{2}} e^{T} P L L^{T} P e \leq \frac{1}{2} \rho^{2} \tilde{d}^{T} \tilde{d}
$$

Eq. (36) is substituted in Eq. (33) and the inequality is enforced, thus giving 


$$
\dot{V} \leq-\frac{1}{2} e^{T} Q e+\frac{1}{2} \rho^{2} \tilde{d}^{T} \tilde{d}
$$

Eq. (37) shows that the $H_{\infty}$ tracking performance criterion is satisfied. The integration of $\dot{V}$ from 0 to $T$ gives

$$
\begin{gathered}
\int_{0}^{T} \dot{V}(t) d t \leq-\frac{1}{2} \int_{0}^{T}\|e\|_{Q}^{2} d t+\frac{1}{2} \rho^{2} \int_{0}^{T}\|\tilde{d}\|^{2} d t \Rightarrow \\
2 V(T)+\int_{0}^{T}\|e\|_{Q}^{2} d t \leq 2 V(0)+\rho^{2} \int_{0}^{T}\|\tilde{d}\|^{2} d t
\end{gathered}
$$

Moreover, if there exists a positive constant $M_{d}>0$ such that $\int_{0}^{\infty}\|\tilde{d}\|^{2} d t \leq M_{d}$, then one gets

$$
\int_{0}^{\infty}\|e\|_{Q}^{2} d t \leq 2 V(0)+\rho^{2} M_{d}
$$

Thus, the integral $\int_{0}^{\infty}\|e\|_{Q}^{2} d t$ is bounded. Furthrmore, $V(T)$ is bounded and from the definition of the Lyapunov function $V$ in Eq. (27) it becomes clear that $e(t)$ will be also bounded since $e(t) \in \Omega_{e}=\left\{e \mid e^{T} P e \leq 2 V(0)+\rho^{2} M_{d}\right\}$. According to the above and with the use of Barbalat's Lemma one obtains $\lim _{t \rightarrow \infty} e(t)=0$.

\section{Simulation tests}

The obtained simulation results are depicted in Fig. 3 to Fig. 4. The real values of the state vector elements of the propulsion system are printed in blue, the estimated values (which have been obtained with the use of the H-infinity Kalman Filter) are printed in green, while the reference setpoints are printed in red. It can be observed that the nonlinear optimal control method achieved fast and accurate tracking of the reference setpoints. It can be also confirmed that the amplitude of the control inputs was moderate. This is important for assuring also moderate energy consumption for the implementation of the control scheme. The estimation of the of the propulsion system's state vector was provided by the H-infinity Kalman Filter after processing a small number of sensor measurements. Actually, to estimate the entire state vector it was necessary to measure only the turn angle of the propeller $x_{1}=\theta_{p}$, the turn angle of the rotor of the induction motor $x_{3}=\theta_{m}$ and the currents of the stator of the induction machine, namely $i_{s d}$ and $i_{s q}$.
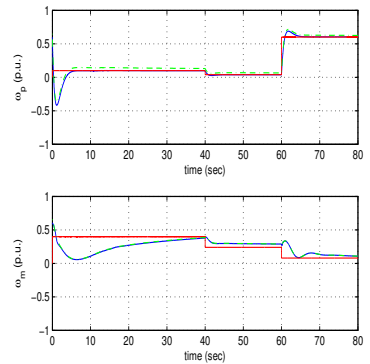

(a)

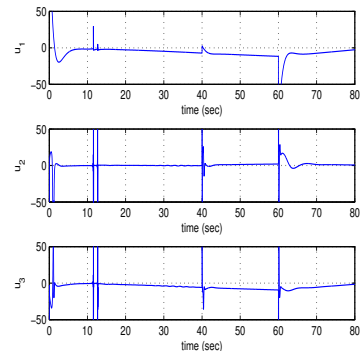

(b)

Figure 3. Tracking performance of the electric ship propulsion system in case of setpoint 1: (a) Convergence of the rotational speed of the propeller $\omega_{p}$ and of the rotational speed of the induction motor to the reference setpoints, (b) Control inputs $u_{1}, u_{2}$ and $u_{3}$ applied to the propulsion system 

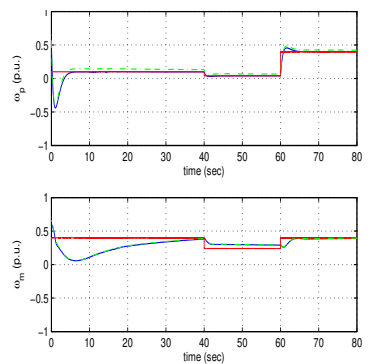

(a)
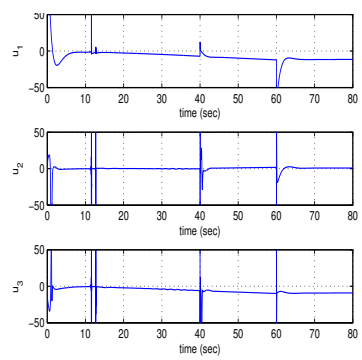

(b)

Figure 4. Tracking performance of the electric ship propulsion system in case of setpoint 2: (a) Convergence of the rotational speed of the propeller $\omega_{p}$ and of the rotational speed of the induction motor to the reference setpoints, (b) Control inputs $u_{1}, u_{2}$ and $u_{3}$ applied to the propulsion system

\section{Conclusions}

A nonlinear optimal (H-infinity) control method has been developed for electric ship propulsion systems, comprising a three-phase induction motor, a drivetrain and a propeller. The dynamic model of the propulsion system has undergone approximate linearization around a temporary operating point that was redefined at each iteration of the control method. The linearization procedure relied on Taylor series expansion and on the computation of Jacobian matrices. For the approximately linearized model of the propulsion system, an optimal (H-infinity) feedback control has been designed. This control method represents the solution to a min-max differential game in which the controller tries to minimize a quadratic cost function of the state vector's error whereas the model uncertainty and external perturbation terms try to maximize this cost function. The stability properties of the control scheme have been proven through Lyapunov analysis.

Acknowledgement: Part of this work that was performed at ISI/ATHENA was supported by the project "I3T - Innovative Application of Industrial Internet of Things (IIoT) in Smart Environments" (MIS 5002434) which is implemented under the "Action for the Strategic Development on the Research and Technological Sector", funded by the Operational Programme "Competitiveness, Entrepreneurship and Innovation" (NSRF 2014-2020) and co-financed by Greece and the European Union (European Regional Development Fund. Besides, part of the collaborative work in this paper was achieved through the 2016-2019 Research Cooperation agreement between the Unit of Industrial Automation of the Industrial Systems Institute and the Nonlinear Control Group of the University of Northumbria, under the financial support of the British University.

\section{References}

[1] J.L.Kirtley, A. Banerjee and S. Englebretson, Motors for ship propulsion, Proceedings of IEEE, 103, 12 (2015).

[2] P. Stone, D.F. Opilo, H. Park, J. Sun, S. Pekarek, R. deCarlo, E. Westervelt, J. Brooks and G. Seenuman, Shipboard Power Management Using Constrained Nonlinear Model 
Predictive Control, IEEE ESTS 2015, IEEE Electric Ship Technology Symposium, (2015), Alexandria, Virginia, USA.

[3] A. Banerjee, M.S. Tomovich, S.B. Loob and J.L. Kirtley, Control application for a switched doubly-fed machine propulsion drive, IEEE Transactions on Industry Applications, 51, 2 (2015).

[4] H. Zahr, F. Seuiller and E. Semail, Five-phase version 12 slots / 8 poles three-phase synchronous machine for marine propulsion, IEE Vehicle Power and Propulsion Conference, Portugal (2014).

[5] D. Zito, C. Bruzzece, E. Santini, A. Raimo and A. Tessarolo, Performance and efficiency improvement of a hydraulic ship steering gear by a Permanent Magnet Linear Synchronous servo-motor, IEEE ICRERA 2015, Intl. Conference on Renewable Energy Research and Applications, Palermo, Italy (2016).

[6] J. Kraszewski, Ship's propulsion neural controller main engine - pitch propeller - shaft generator, IFAC 3rd Intl. Conference of Advances in Control and Optimization of Dynamical Systems, Kanpur, India, (2014).

[7] E. Skjang, J.A. Suul, A. Rygg, J.A. Johansen and M. Molinas, System-wide harmonic mitigation in a diesel electric ship by Model Predictive Control, IEEE Transactions on Industrial Electronics, 63, 7 (2016).

[8] R.T. Geertsma, R.R. Negenborn, K. Visser and J.J. Hopmann, Parallel control for hybrid propulsion of multi-function ships, 20th IFAC World Congress, Toulous, France, (2007).

[9] S.Y. Kim, S. Choe, S. Lo, S. Kim and S.K. Sul, Electric porpulsion novel ships with energy storage models through AFE and Converters, Journal of Power Electronics, 14, 2 (2014).

[10] J. Hu, J. Sun and H.F. Hofmann, Mitigating power fluctuations in electric ship propulsion with hybrid energy storage system: Design and analysis, IEEE Journal of Oceanic Engineering, (2017).

[11] S.H. Kia, H. Henae and C.A. Capolino, Gear tooth surface damage fault detection using induction machine stator current space vector analysis, IEEE Transactions on Industrial Electronics, 62 3, (2015).

[12] G. Rigatos, Intelligent Renewable Energy Systems: Modelling and Control, Springer, (2016).

[13] G. Rigatos, P. Siano, P. Wira and F. Profumo, Nonlinear H-infinity Feedback Control for Asynchronous Motors of Electric Trains, Journal of Intelligent Industrial Systems, Springer, (2015)

[14] G.G. Rigatos and S.G. Tzafestas, Extended Kalman Filtering for Fuzzy Modelling and Multi-Sensor Fusion, Mathematical and Computer Modelling of Dynamical Systems, Taylor \& Francis , 13 (2007).

[15] M. Basseville and I. Nikiforov, Detection of abrupt changes: Theory and Applications, Prentice-Hall (1993).

[16] G. Rigatos and Q. Zhang, Fuzzy model validation using the local statistical approach, Fuzzy Sets and Systems, Elsevier, 60, 7 (2009).

[17] G. Rigatos, Nonlinear control and filtering using differential flatness approaches: applications to electromechanicsl systems, Springer (2015).

[18] G.J. Toussaint, T. Basar and F. Bullo, $H_{\infty}$ optimal tracking control techniques for nonlinear underactuated systems, in Proc. IEEE CDC 2000, 39th IEEE Conference on Decision and Control, Sydney Australia (2000). 